\title{
Supplement for "Intercomparison of upper tropospheric and lower stratospheric water vapor measurements over the Asian Summer Monsoon during the StratoClim Campaign"
}

Clare E. Singer ${ }^{1, \mathrm{a}}$, Benjamin Clouser ${ }^{1}$, Sergey Khaykin ${ }^{2}$, Martina Krämer $^{3}$, Francesco Cairo ${ }^{4}$, Thomas Peter ${ }^{5}$, Alexey Lykov ${ }^{6}$, Christian Rolf ${ }^{3}$, Nicole Spelten ${ }^{3}$, Simone Brunamonti ${ }^{5, b}$, and Elisabeth J. Moyer ${ }^{1}$

${ }^{1}$ Dept. of the Geophysical Sciences, University of Chicago, Chicago, IL, USA

${ }^{2}$ LATMOS, UVSQ, Sorbonne Université, CNRS, IPSL, Guyancourt, France

${ }^{3}$ Forschungszentrum Jülich, Institut für Energie und Klimaforschung (IEK-7), Germany

${ }^{4}$ National Research Council of Italy, Institute of Atmospheric Sciences and Climate (CNR-ISAC), Rome, Italy

${ }^{5}$ Institute for Atmospheric and Climate Science, ETH Zürich, Zürich, Switzerland

${ }^{6}$ Central Aerological Observatory of RosHydroMet, Dolgoprudny, Russian Federation

${ }^{a}$ now at: Dept. of Environmental Science and Engineering, California Institute of Technology, Pasadena, CA, USA

${ }^{b}$ now at: Empa, Laboratory for Air Pollution/Environmental Technology, Dübendorf, Switzerland

Correspondence: C. E. Singer (csinger@caltech.edu) and E. J. Moyer (moyer@uchicago.edu) 
Flight 2

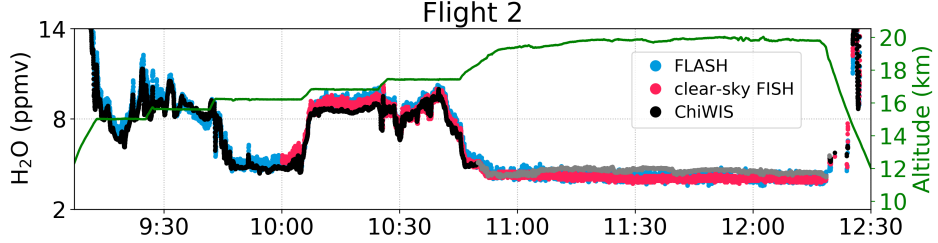

Flight 3

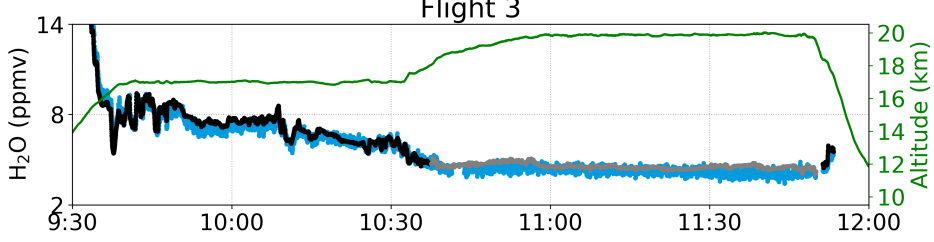

Flight 4

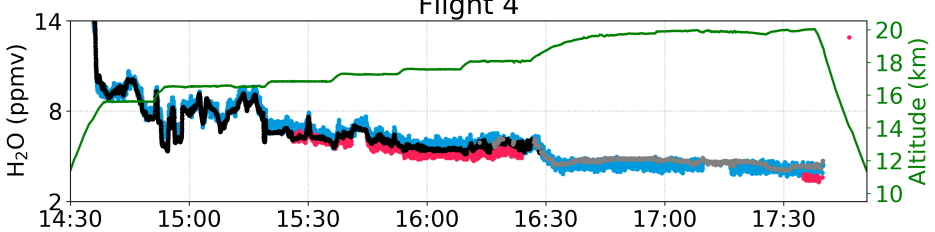

Flight 6
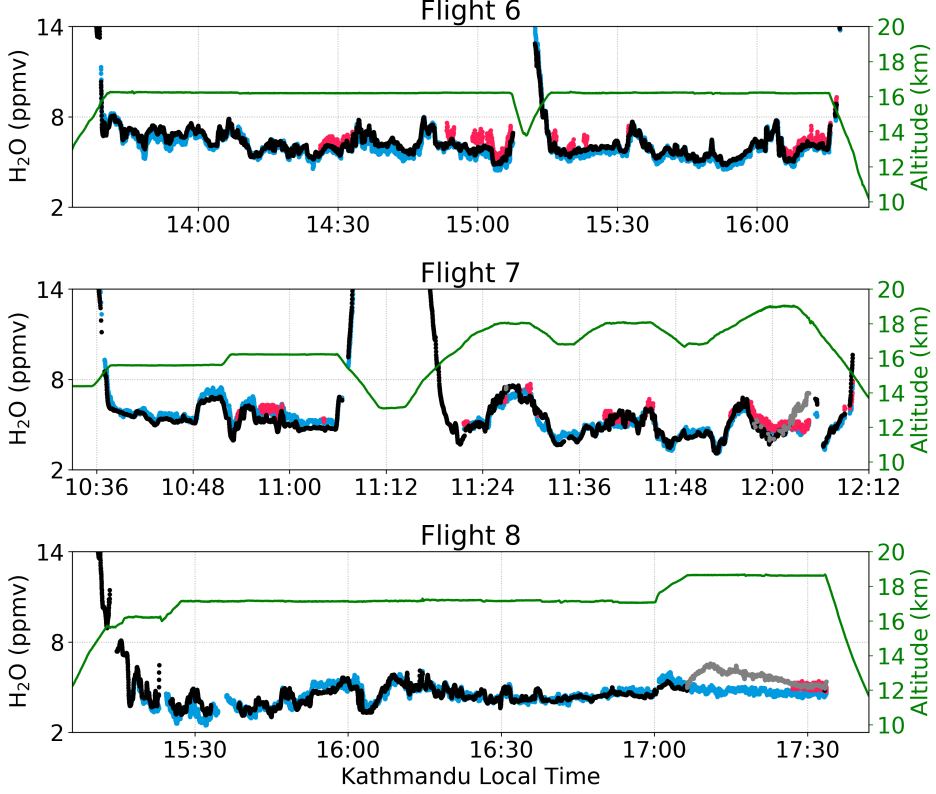

Figure S1. Time series of water vapor for each of the six flights (F2-F4 and F6-F8). Altitude (green) and in situ $\mathrm{H}_{2} \mathrm{O}$ measurements from ChiWIS (black/grey), FLASH (blue), and clear-sky FISH (pink). Periods where ChiWIS cell pressure is just out of regulation (20-30 mbar), are shown in grey. There is impressive agreement between the three hygrometers. ChiWIS shows much higher precision that the other two instruments, which is evident from the sharper temporal structures in the time series. Nevertheless, all three instruments are able to capture the same fine-scale variability of the atmosphere. 
Flight 2

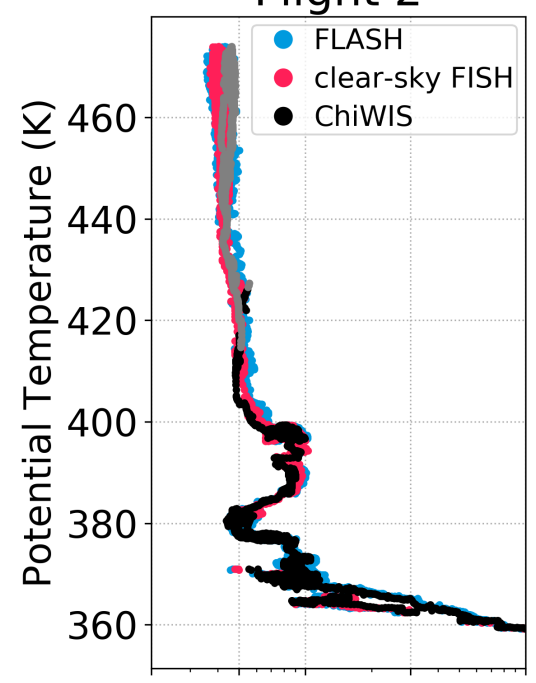

Flight 6

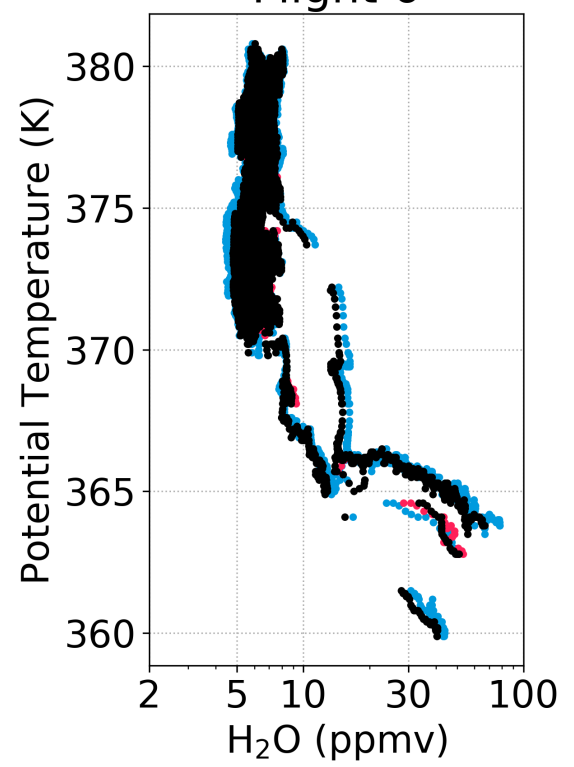

Flight 3

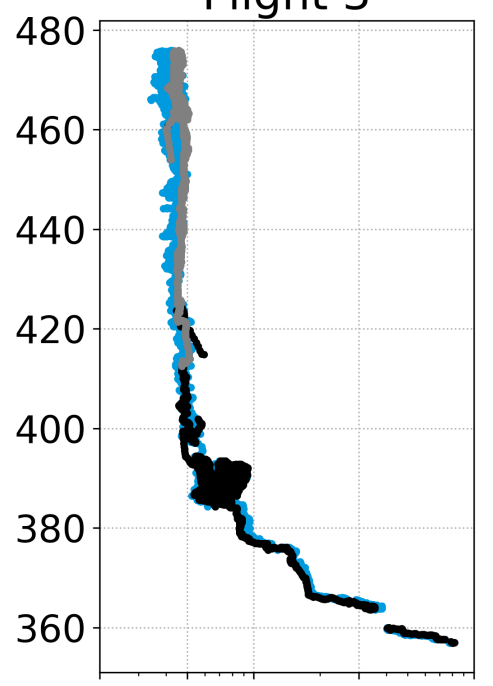

Flight 7

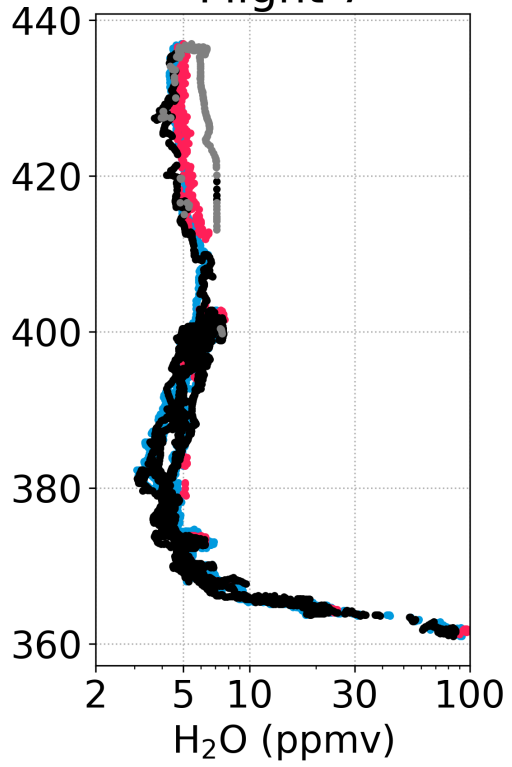

Flight 4

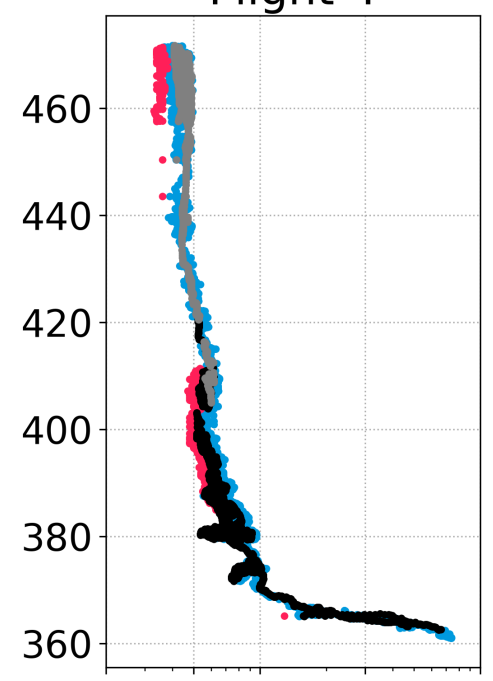

Flight 8

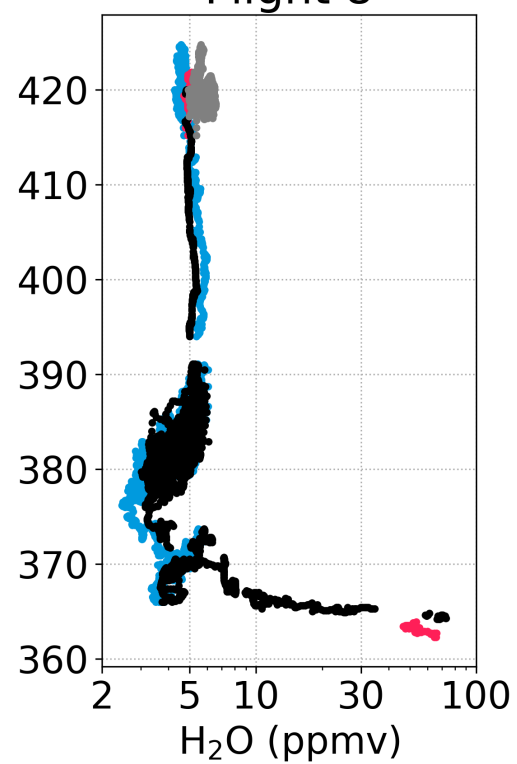

Figure S2. Vertical profiles of water vapor against potential temperature for each of the six flights (F2-F4 and F6-F8). Shown are in situ $\mathrm{H}_{2} \mathrm{O}$ measurements from ChiWIS (black/grey), FLASH (blue), and clear-sky FISH (pink), with periods where ChiWIS cell pressure is just out of regulation $(20-30$ mbar) shown in grey. 
Flight 2

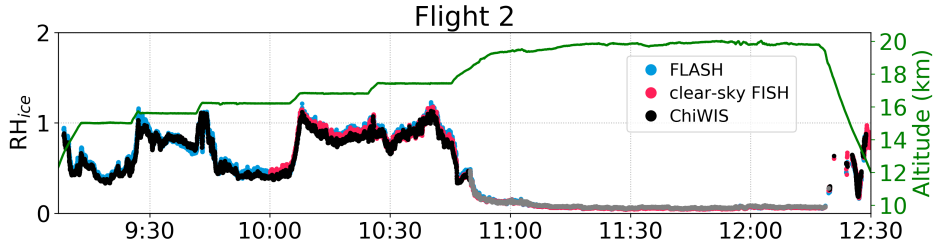

Flight 3

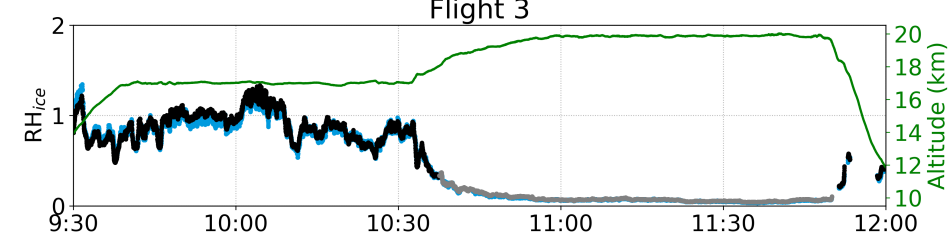

Flight 4

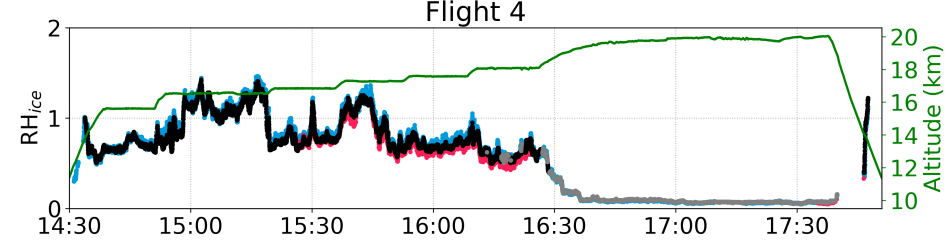

Flight 6

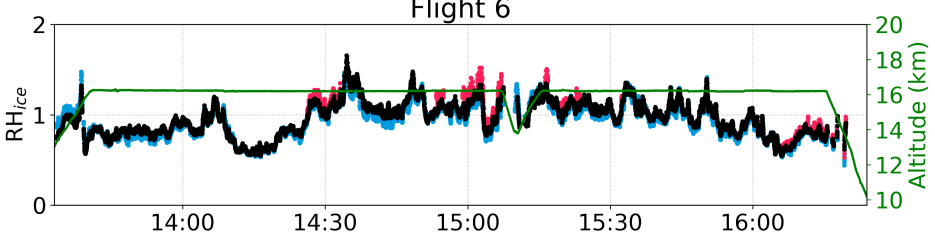

Flight 7

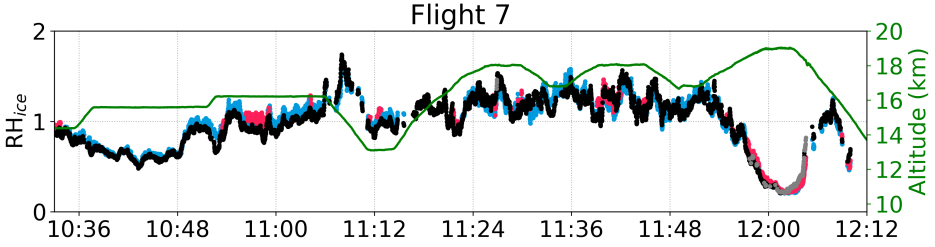

Flight 8

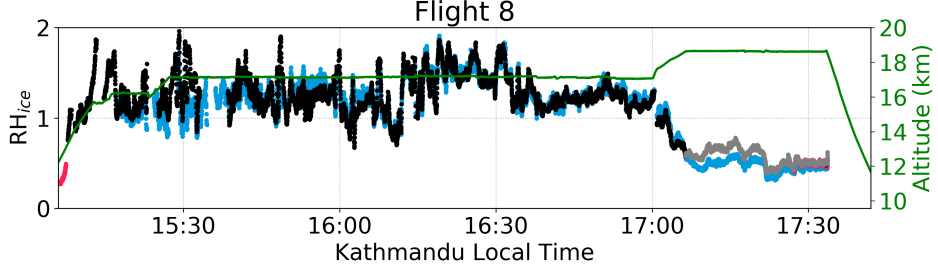

Figure S3. Time series of relative humidity for each of the six flights (F2-F4 and F6-F8). Shown are altitude (green) and in situ RH $\mathrm{ice}$ measurements from ChiWIS (black/grey), FLASH (blue), and clear-sky FISH (pink) with periods where ChiWIS cell pressure is just out of regulation $(20-30$ mbar) shown in grey. 


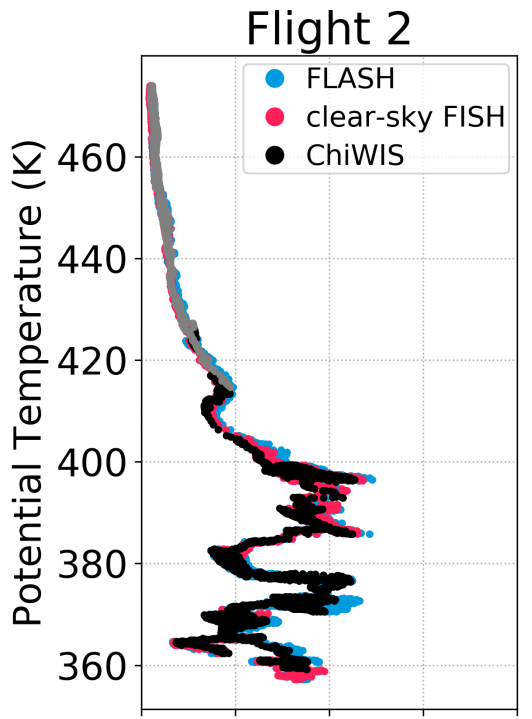

Flight 6

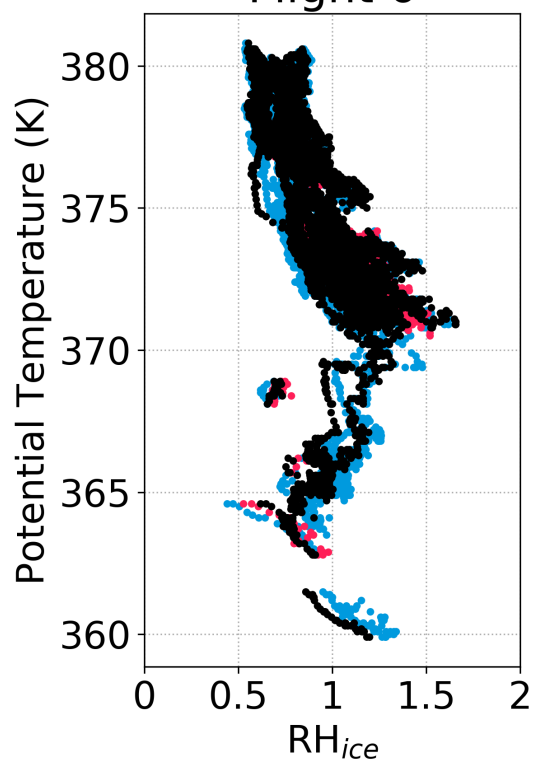

Flight 3

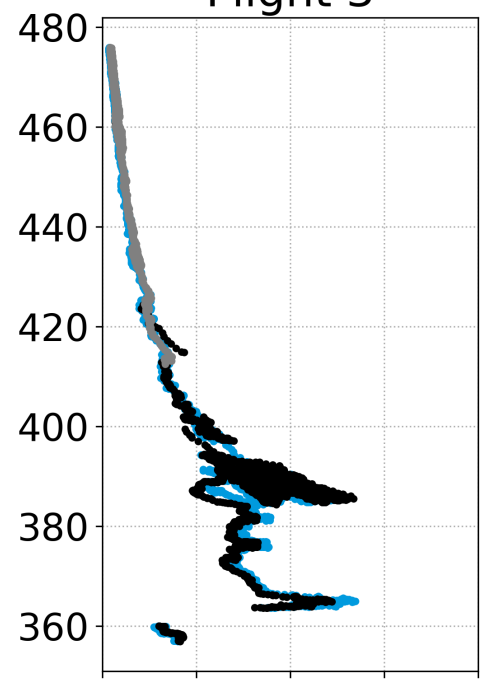

Flight 7

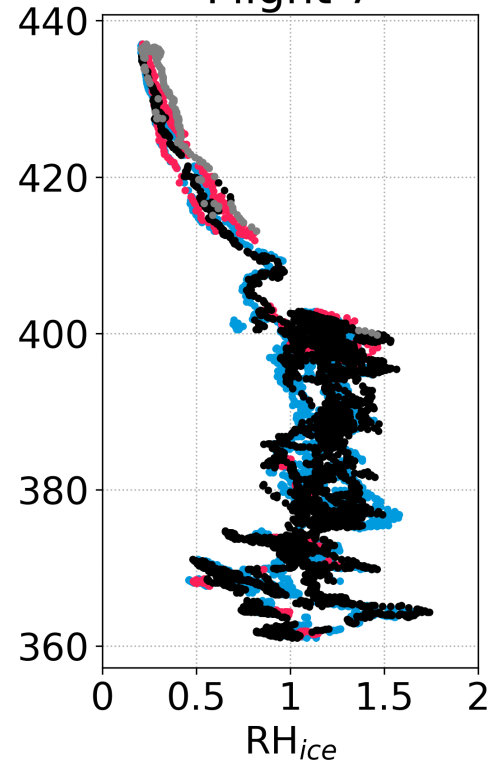

Flight 4

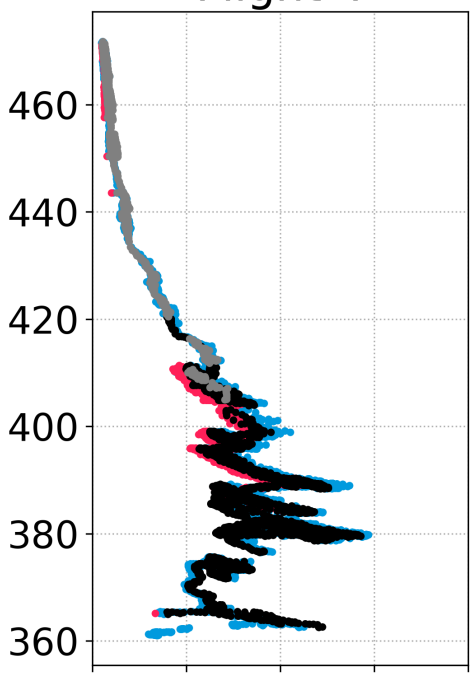

Flight 8

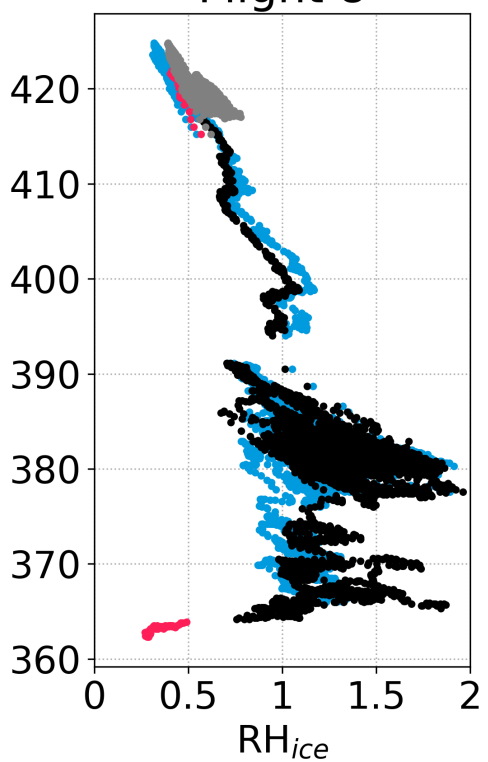

Figure S4. Vertical profiles of relative humidity against potential temperature for each of the six flights (F2-F4 and F6-F8). Shown are in situ $\mathrm{RH}_{\text {ice }}$ measurements from ChiWIS (black/grey), FLASH (blue), and clear-sky FISH (pink) with periods where ChiWIS cell pressure is just out of regulation $(20-30$ mbar) shown in grey. 


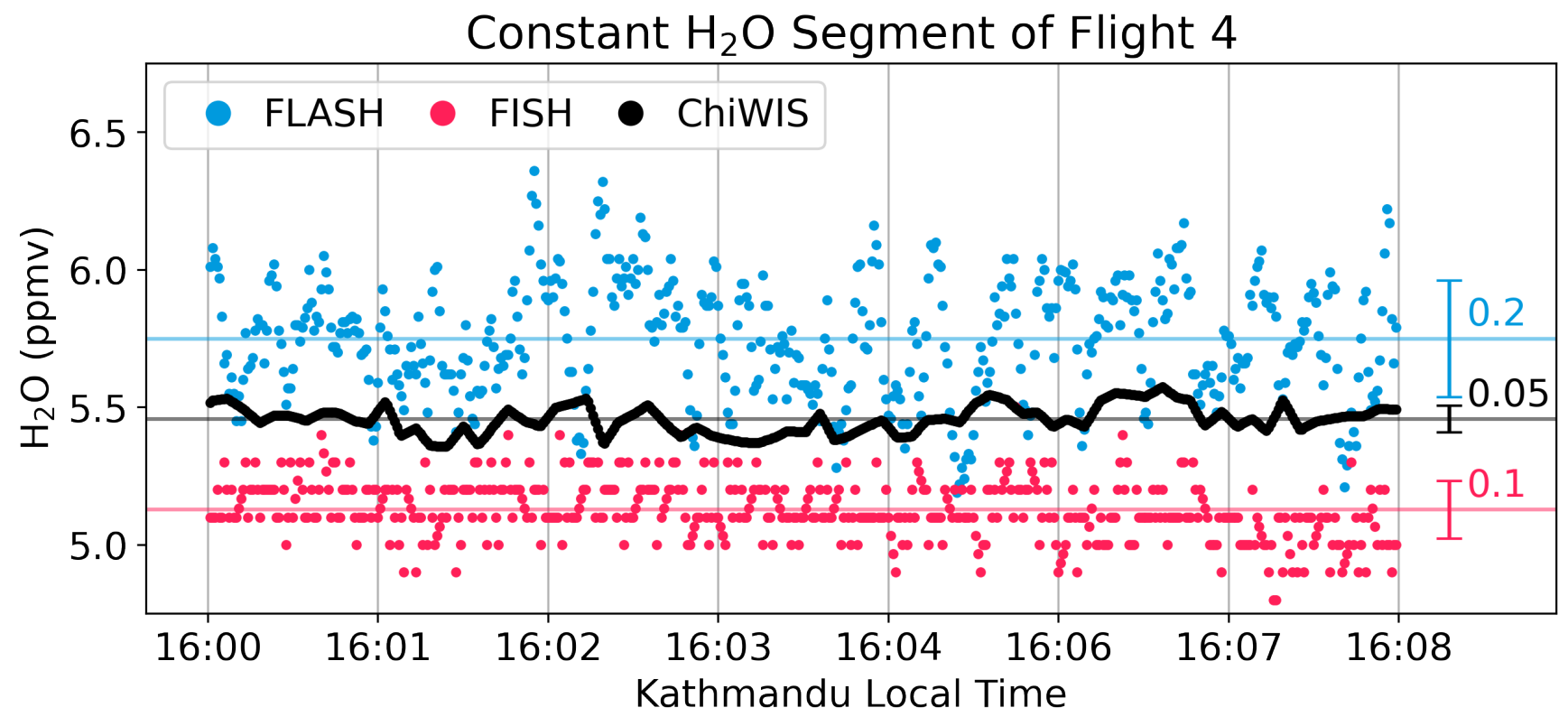

Figure S5. Estimated in-flight precision of the three hygrometers based on a a segment from flight 4 with approximately constant $\mathrm{H}_{2} \mathrm{O}$. Shown in colored dots are the $\mathrm{H}_{2} \mathrm{O}$ values measured by FLASH, FISH, and ChiWIS (blue, pink, black), the mean value for this period (transparent lines), and the standard deviation of the measurements (plotted error bars with labeled values). 


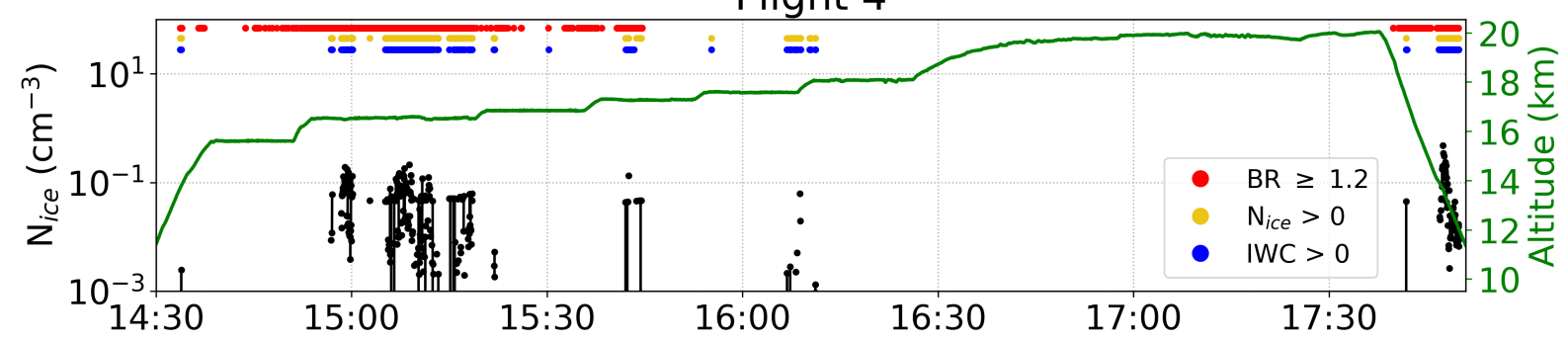

Flight 6

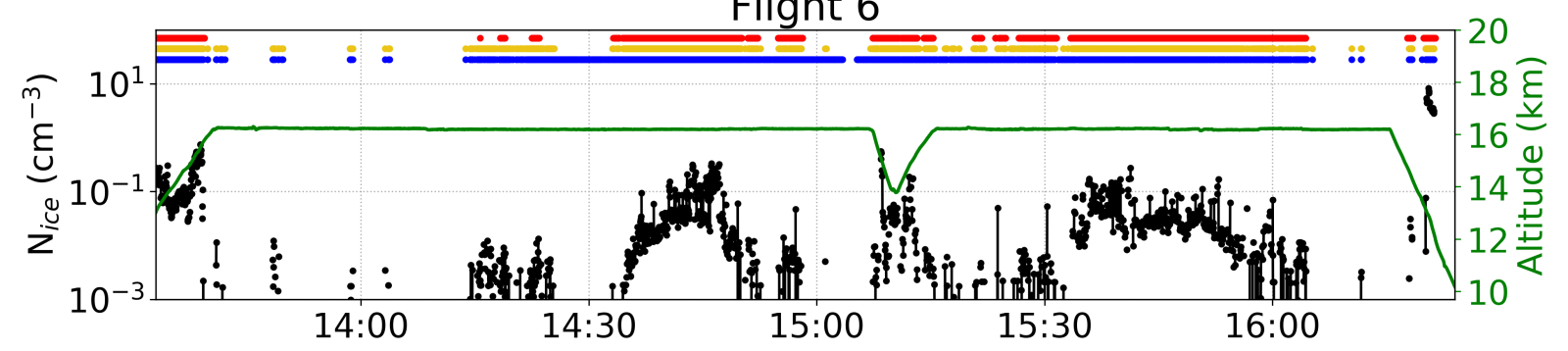

Flight 7

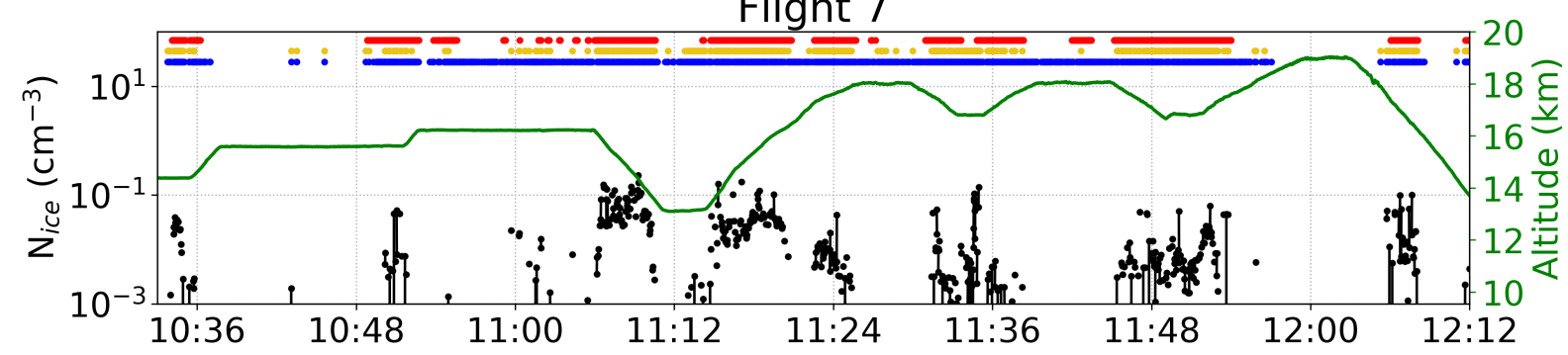

Flight 8

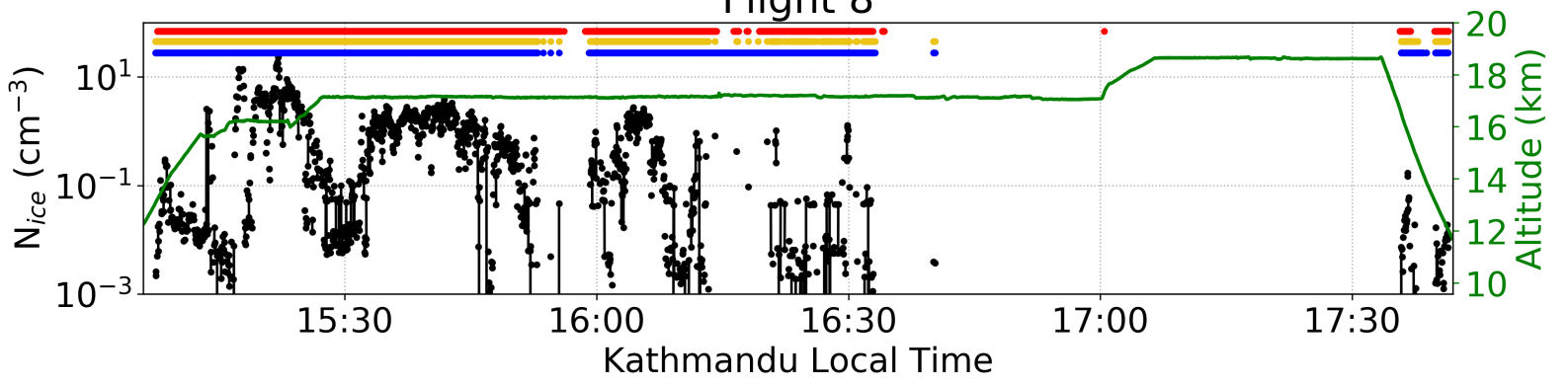

Figure S6. Time series of ice particle number concentration $\left(\mathrm{N}_{\mathrm{ice}}\right.$, black) measured by NIXE-CAPS for F4, F6, F7, and F8. No clouds were sampled on F2 or F3. Shown in colors at the top of each panel are cloud flags derived from MAS backscatter ratio (BR $\geq 1.2$, red), $\mathrm{N}_{\text {ice }}>0 \mathrm{~cm}^{-3}$ (yellow), and ice water content produce (IWC $>0 \mathrm{~g} \mathrm{~kg}^{-1}$, blue). Clear-sky is inversely defined as regions where BR $<1.2$ or $\mathrm{N}_{\text {ice }}=0 \mathrm{~cm}^{-3}$. The IWC is defined as the difference between FISH and FLASH, and is thus an unreliable flag for clear-sky in an intercomparison study like this where we are specifically interested in the small deviations in vapor measurements between these two instruments. Flight altitude is shown in green. 

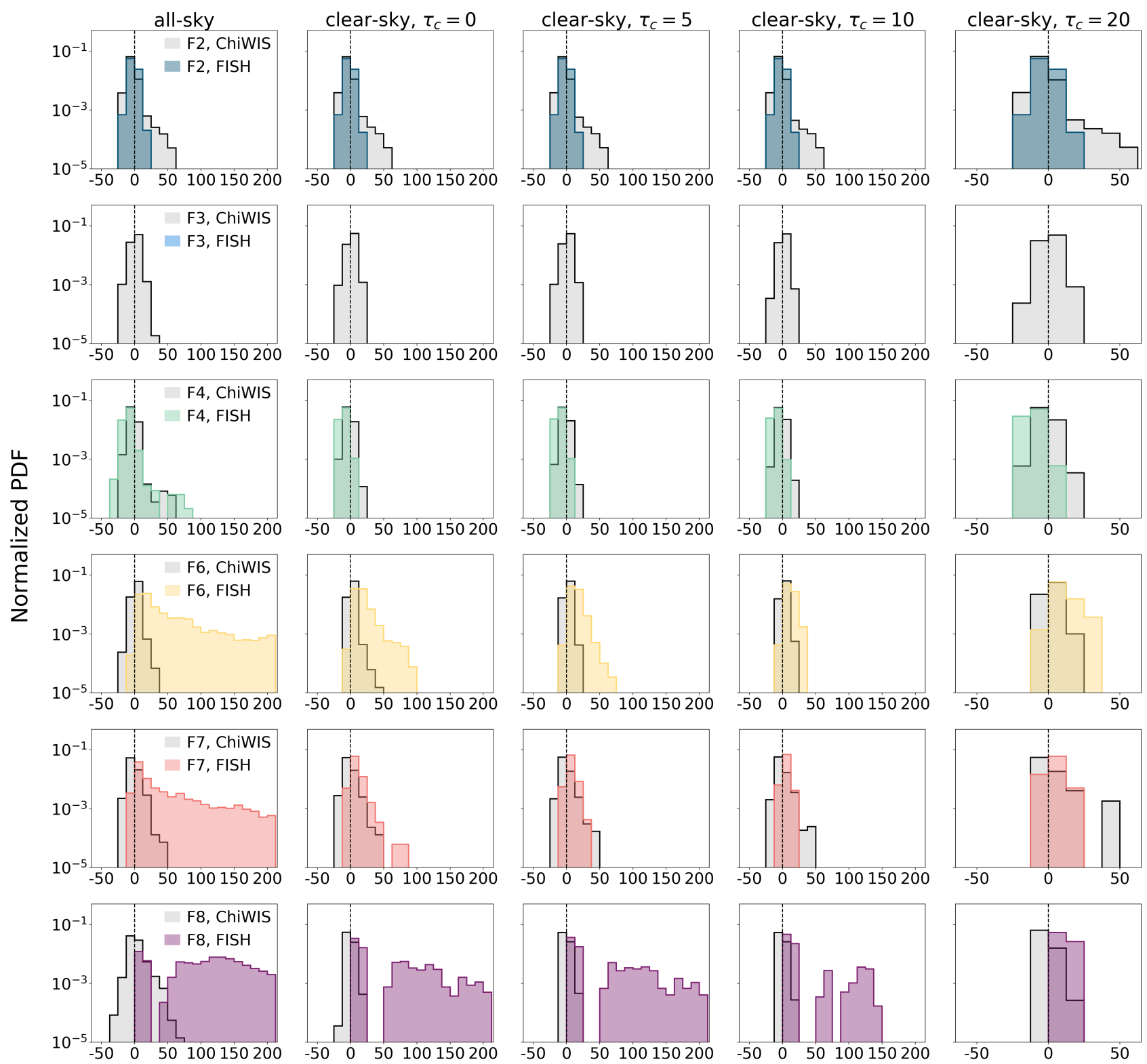

$\%$ diff from FLASH

Figure S7. Normalized PDF of percentage differences between ChiWIS (grey) and FISH (colors) from FLASH during each flight (rows). FISH did not report measurements on F3. The first column (leftmost) shows values from all conditions. The second column shows percentage difference in clear-sky periods with zero time lag, $\tau_{c}=0$. Clear-sky is defined as regions where $\mathrm{BR}<1.2 \mathrm{or} \mathrm{N} \mathrm{ice}=0 \mathrm{~cm}{ }^{-3}$. Each subsequent column shows the percentage difference in clear-sky periods with increasing $\tau_{c}=5,10,20$. Note that the last column (rightmost) has a smaller $\mathrm{x}$-axis range. 


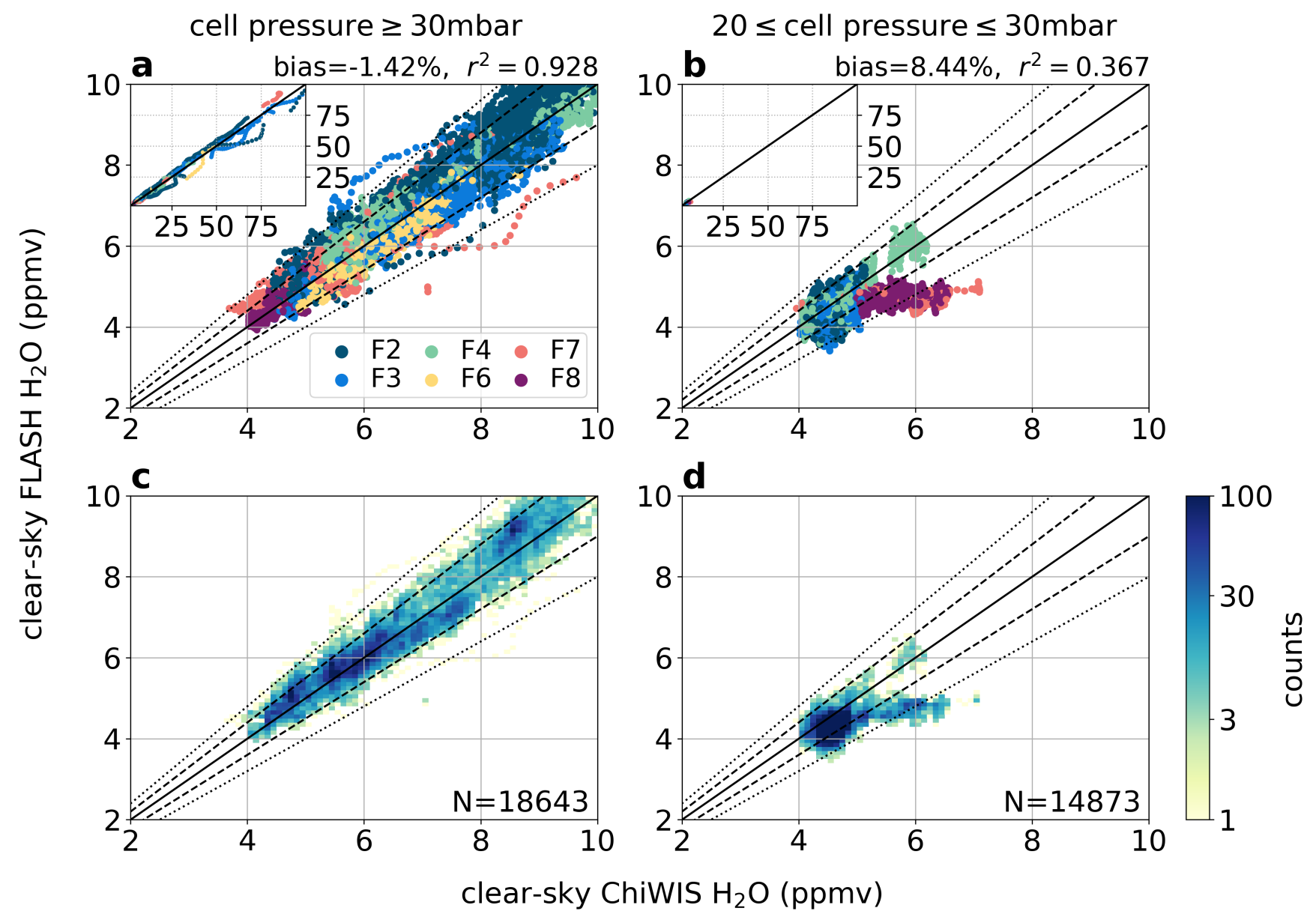

Figure S8. The left column (a,c) reproduces Fig. 2b,e showing a point-by-point comparison of clear-sky $\mathrm{H}_{2} \mathrm{O}$ measured by ChiWIS and FLASH during periods when the ChiWIS optical cell pressure is $\geq 30$ mbar (well regulated). The right column (b,d) shows the same comparison between clear-sky ChiWIS and FLASH measurements, but exclusively for periods when the ChiWIS optical cell pressure is slightly unregulated (between 20 and 30 mbar). Points in (a,b) are colored by flight number. Frequency of counts (c,d) in each 0.1 by $0.1 \mathrm{ppmv}$ bin are shown in color. The numbers in the bottom right corners of each panel indicate the number of measurements in each category. For clear-sky periods, $56 \%$ of measurements were made when the cell pressure was within regulation. For in-cloud periods, nearly $100 \%$ of measurements were made when the cell pressure was within regulation (not shown). The one-to-one line is plotted in solid black with dashed (dotted) lines indicated $\pm 10 \%( \pm 20 \%)$ differences. The percentage bias and $r^{2}$ coefficients are shown above each panel. 

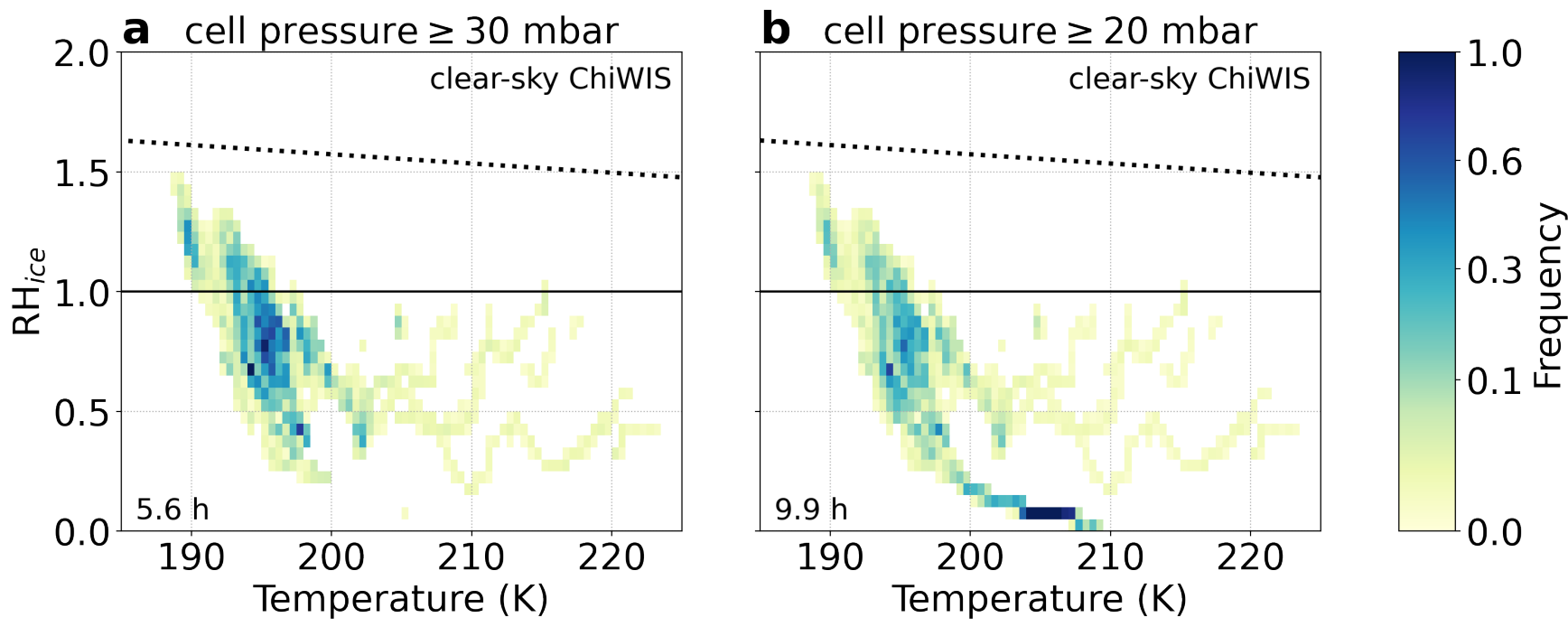

Figure S9. Normalized frequency distribution of ChiWIS measurements in clear-sky at different temperatures and relative humidities. (a) Reproduces Fig. 6a, showing measurements when the ChiWIS optical cell pressure is $\geq 30$ mbar. (b) Shows measurements when the cell pressure is $\geq 20$ mbar. The measurements are binned by $0.5 \mathrm{~K}$ in temperature and 0.05 in relative humidity. The number of individual measurements is shown in the bottom right of each panel. The solid black lines shows $\mathrm{RH}_{\mathrm{ice}}=1$ and the dotted line shows the homogeneous ice nucleation threshold from Koop et al. (2000). 


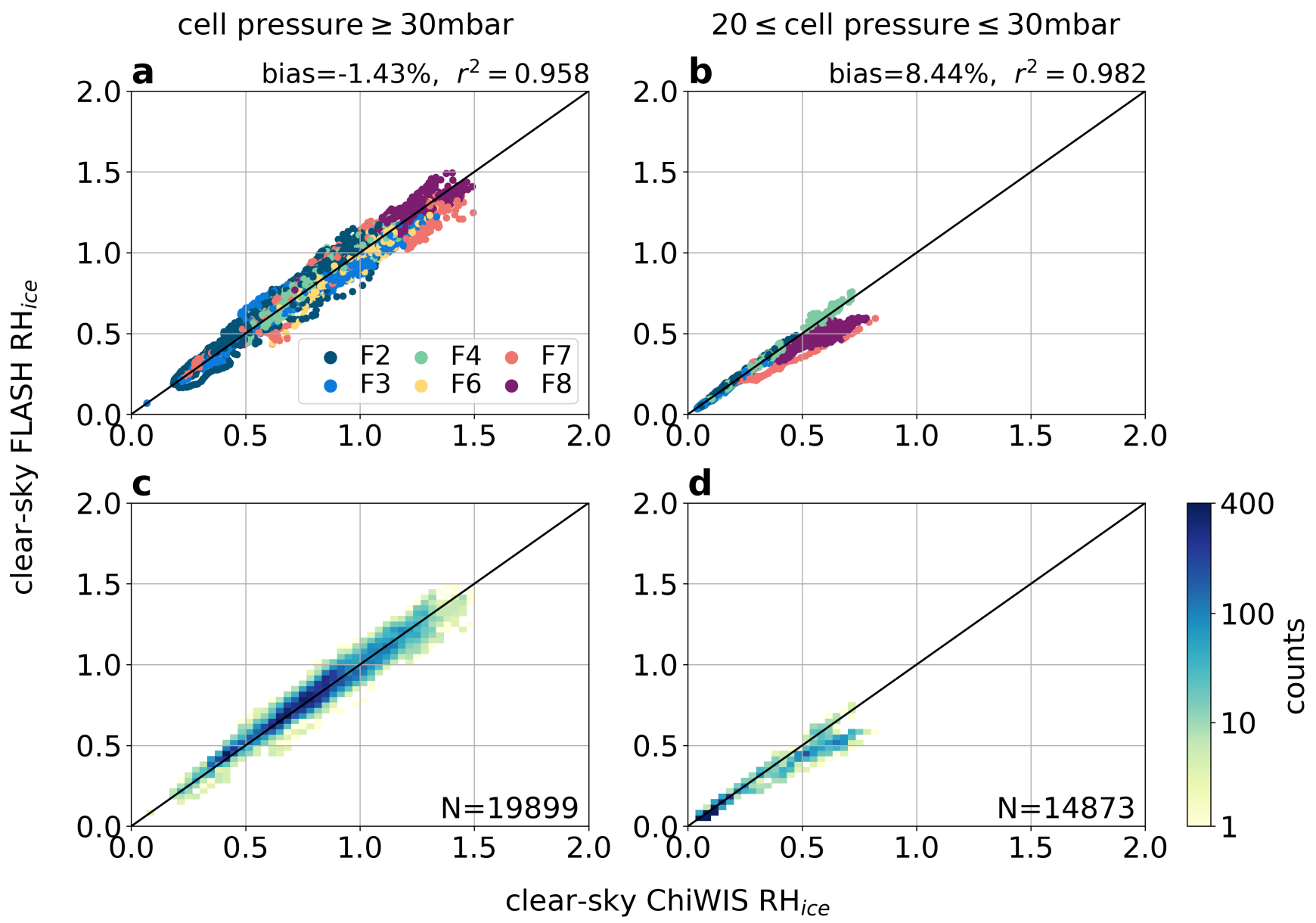

Figure S10. The left column $(\mathrm{a}, \mathrm{c})$ reproduces Fig. 7b,e showing a point-by-point comparison of clear-sky $\mathrm{RH}_{\mathrm{ice}}$ measured by ChiWIS and FLASH during periods when the ChiWIS optical cell pressure is $\geq 30$ mbar (well regulated). The right column (b,d) shows the same comparison between clear-sky ChiWIS and FLASH measurements, but exclusively for periods when the ChiWIS optical cell pressure is slightly unregulated (between 20 and 30 mbar). Points in (a,b) are colored by flight number. Frequency of counts (c,d) in each 0.03 by 0.03 bin are shown in color. The numbers in the bottom right corners of each panel indicate the total hours of measurement. For clear-sky periods, $56 \%$ of measurements were made when the cell pressure was within regulation. For in-cloud periods, nearly $100 \%$ of measurements were made when the cell pressure was within regulation (not shown). The one-to-one line is plotted in black. The percentage bias and $r^{2}$ coefficients are shown above each panel. 


\section{References}

Koop, T., Luo, B., Tsias, A., and Peter, T.: Water activity as the determinant for homogeneous ice nucleation in aqueous solutions, Nature, 406, 611-614, https://doi.org/10.1038/35020537, 2000. 